\title{
Superconducting magnetic energy storage and superconducting self-supplied electromagnetic launcher ${ }^{\star}$
}

\author{
Jérémie Ciceron* , Arnaud Badel, and Pascal Tixador \\ Institut Néel, G2ELab CNRS/Université Grenoble Alpes, Grenoble, France
}

Received: 5 December 2016 / Received in final form: 8 April 2017 / Accepted: 16 August 2017

\begin{abstract}
Superconductors can be used to build energy storage systems called Superconducting Magnetic Energy Storage (SMES), which are promising as inductive pulse power source and suitable for powering electromagnetic launchers. The second generation of high critical temperature superconductors is called coated conductors or REBCO (Rare Earth Barium Copper Oxide) tapes. Their current carrying capability in high magnetic field and their thermal stability are expanding the SMES application field. The BOSSE (Bobine Supraconductrice pour le Stockage d'Energie) project aims to develop and to master the use of these superconducting tapes through two prototypes. The first one is a SMES with high energy density. Thanks to the performances of REBCO tapes, the volume energy and specific energy of existing SMES systems can be surpassed. A study has been undertaken to make the best use of the REBCO tapes and to determine the most adapted topology in order to reach our objective, which is to beat the world record of mass energy density for a superconducting coil. This objective is conflicting with the classical strategies of superconducting coil protection. A different protection approach is proposed. The second prototype of the BOSSE project is a small-scale demonstrator of a Superconducting Self-Supplied Electromagnetic Launcher (S3EL), in which a SMES is integrated around the launcher which benefits from the generated magnetic field to increase the thrust applied to the projectile. The S3EL principle and its design are presented.
\end{abstract}

\section{Motivation}

\subsection{SMES principle [1]}

Superconductors have the property to lose their electrical resistivity when they are cooled under a critical temperature $T_{C}$. Even if energy dissipation occurs in a superconductor submitted to variable electrical current or magnetic induction $(B)$, there is no energy dissipation in a steady state. If a superconducting winding is supplied, then short-circuited current is not dissipated by Joule effect and magnetic energy is conserved almost indefinitely. This is the principle of inductive storage with superconductors, generally called SMES (Superconducting Magnetic Energy Storage).

The stored energy $E_{\text {mag }}$ can be expressed as a function of inductance $L$ and current $I$ or as the integral over space of the product of magnetic field $H$ by induction $B$, following (1):

$$
E_{\text {mag }}=\frac{1}{2} L I^{2}=\frac{1}{2} \iiint_{\text {Space }} B H d x d y d z .
$$

Once the SMES has been charged and short-circuited, the energy is available and can be used by opening the short circuit and connecting the SMES to a load. It can be connected to the load either directly as in the case of direct

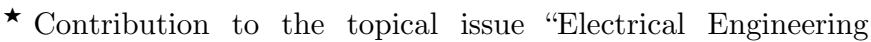
Symposium (SGE 2016)", edited by Adel Razek

* e-mail: jeremie.ciceron@gelab.grenoble-inp.fr
}

supply of an electromagnetic launcher, or through a voltage adaptation system if the discharge has to be controlled. SMES have low energy density compared to batteries, but high power densities. Furthermore, they can have high cycling yield (97\%), with the cycling yield being defined as the recovered energy divided by the energy provided to the SMES, including the energy spent to cool the system, after one cycle. They are direct electricity storage devices such as capacitors. Nevertheless, they can have much higher energy density than high power capacitor banks. The volume energy of a SMES can be roughly estimated if the $B$ field is considered homogeneous in the SMES and if the winding thickness is neglected. In these conditions, equation (1) gives a volume energy of $32 \mathrm{MJ} / \mathrm{m}^{3}$ for a SMES having a homogeneous field of $9 \mathrm{~T}$, and $57 \mathrm{MJ} / \mathrm{m}^{3}$ for $12 \mathrm{~T}$. In comparison, the volume energy of high power capacitor banks is around $1 \mathrm{MJ} / \mathrm{m}^{3}$. Thanks to High Temperature Superconductors (HTS), a SMES with a field of $12 \mathrm{~T}$ or even more is feasible. Second generation HTS conductors, also called coated conductors or REBCO (Rare Earth, Barium, Copper Oxide) tapes, are the most performing superconductors today in terms of critical temperature as well as in terms of engineering current under high magnetic fields. They offer the opportunity to largely improve the performances of SMES in terms of mass, volume, thermal stability and therefore to expand their application field. Even if the critical temperature of these tapes is high $(90 \mathrm{~K})$, they are generally used at $4.2 \mathrm{~K}$ (liquid helium bath) in high field applications because the 


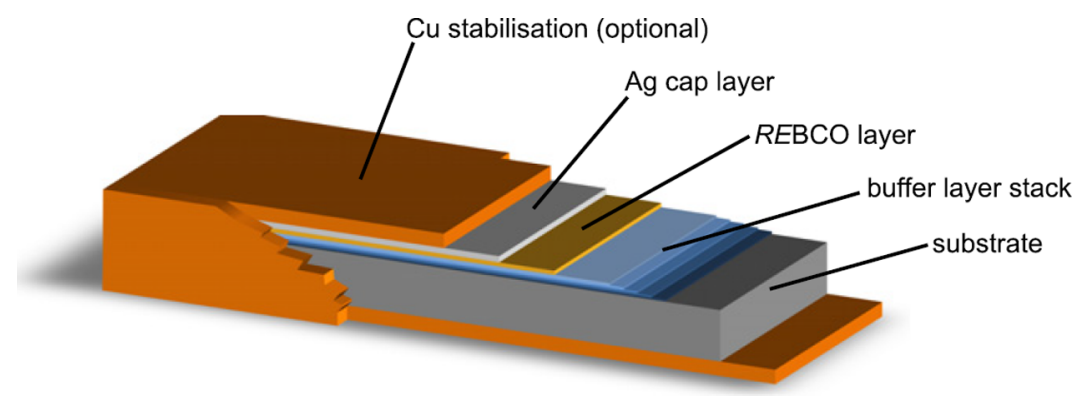

Fig. 1. Schematic layout of REBCO tapes. The layers are not up to scale (courtesy C. Barth, University of Geneva).
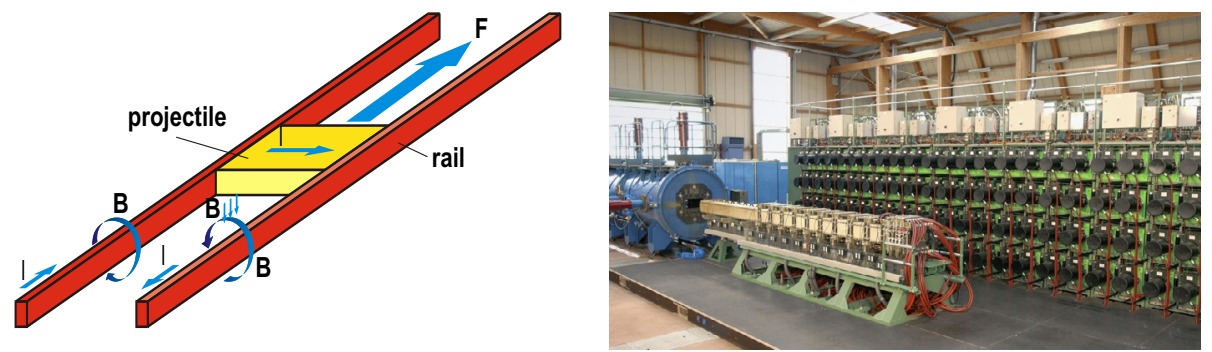

Fig. 2. Principle diagram of an electromagnetic launcher (railgun) and picture of the PEGASUS launcher (10 MJ).

more a superconductor is cooled, the more its current carrying capability is increased. On the contrary, the higher is the $B$ field, the lower is the current carrying capability. REBCO conductors are commercially available in the form of flat tapes (see Fig. 1) which are commonly around $100 \mu \mathrm{m}$ thick; custom thicknesses are availed from some manufacturers.

Combination of modest energy density with very high power density naturally leads to consider SMES as pulse current source or connected to the grid to compensate short power faults [2].

\subsection{Intended application}

Since 2004, a program for the development of SMES has been led at Grenoble CNRS [3] in order to consider HTS SMES as power source to supply electromagnetic launchers. An electromagnetic launcher, commonly called "railgun", is made of two parallel conductive rails, with a projectile establishing a sliding contact between them. A high electrical current passing through the rails and the projectile is accelerating the projectile, according to the same principle as in the famous Laplace rail experiment. The force applied to the projectile is:

$$
\vec{F}=I \vec{\ell} \times \vec{B}
$$

where $\ell$ is the distance between rails, $I$ is the current passing in the projectile and $B$ is the magnetic induction on the projectile.

These launchers (see Fig. 2) can propel projectiles at higher velocities than conventional powder guns and research is ongoing in several countries on this topic $[4,5]$. In the 10 MJ PEGASUS launcher developed at ISL, the projectile can reach an output velocity of $2000 \mathrm{~m} / \mathrm{s}$ for an electrical current of $1 \mathrm{MA}$ [6]. Electromagnetic launchers are generally supplied by capacitor banks. These are very voluminous because of their low energy density on one side, and because of the poor supply chain yield on the other side. A SMES, being an inductive pulse power source, is particularly adapted for supplying directly an electromagnetic launcher with rails [7].

In most of the launchers (i.e. PEGASUS), the induction $B$ is only generated by the current passing in the rails. But it is possible to increase the value of $B$ between the rails thanks to additional windings, parallel to the rails, and then to increase the propulsive thrust applied to the projectile. In this case, the term "augmented launcher" is used.

The S3EL (Superconducting Self-Supplied Electromagnetic Launcher) concept [8] is combining a powering by SMES with the augmented launcher principle. The energy which is required for the launch is stored in racetrack-shaped windings surrounding the rails and then is released in these last ones. The magnetic induction generated by the windings is increasing the propelling thrust and the projectile output velocity. Otherwise, it can reduce the current required to reach a given output velocity. In the end, this concept enables to lighten and to compact the launcher supply chain. Furthermore, the windings being magnetically coupled to the rails, a part of rails magnetisation energy is recovered by the superconducting windings at the end of the shot, improving the launch global yield and reducing the energy dissipated in the muzzle electric arc which happens when the projectile is leaving the rails.

Nevertheless, to make such a device at full scale is technologically very challenging because a launcher nominal current is in the range of several tens or hundreds of $\mathrm{kA}$, and can even be several MA for large size launchers. Even if cables with currents of several $\mathrm{kA}$ have been manufactured and tested under field [9,10], cables with 
currents in the range of hundreds of $\mathrm{kA}$ or more are not existing today. Another solution consists in using the SMES as a buffer power supply in order to quickly recharge a capacitor bank. In this case, the capacitor bank is sized only for one shot [11] but the launcher can fire in bursts.

The objective of the BOSSE project of French Directorate General of Armaments (DGA) is to develop the technology of HTS magnets with very high energy density, foreseeing what could be a buffer energy storage for large size launchers, and to test at low scale the feasibility of a coupled S3EL launcher. This will be achieved through the manufacturing of two prototypes. The first one is a SMES storing a $1 \mathrm{MJ}$ energy with a specific energy of $20 \mathrm{~kJ} / \mathrm{kg}$, more compact and lighter than any existing SMES. The second one is a small scale S3EL electromagnetic launcher ( $1 \mathrm{~m}$ long).

\section{High energy density SMES}

\subsection{Introduction to SMES design}

The objectives of the SMES design are:

- Ensuring the mechanical integrity of the structure and winding. The SMES is submitted to strong mechanical forces. This is fundamentally unavoidable following the Virial theorem [12-14].

- Maintaining the conductor in its superconducting state. In other words, in every point of the winding the nominal current has to be kept under the critical current $I_{C}$. The critical current is the current at which the superconductor is transitioning to normal conducting state, suddenly becoming resistive or even insulating. $I_{C}$ is mainly dependent on temperature, $B$ field value and orientation between $B$ field and tape surface. To a lesser extent, $I_{C}$ is dependent on strain.

- Maximizing the specific energy, since this is the objective of the high energy density SMES from BOSSE project. In our case, specific energy means the total stored energy divided by the total mass of the conductor. The mass of spacers, mandrels, or central trunk in case of the toroid, is not taken into account. These elements are generally in glass fiber reinforced plastic, significantly less dense than the conductor itself which is mainly made of Hastelloy ${ }^{\circledR}$ C-276 and copper (the superconductor layer is only $1 \mu \mathrm{m}$ thick). Furthermore, we will see in Section 2.6 that in our case, lowering the mass of used conductor is equivalent to lowering the required length of conductor and lowering the conductor's total cost. This last criterion is of great interest since the price of REBCO conductors is currently quite high (around $40 € / \mathrm{m}$ for a $4 \mathrm{~mm}$ wide tape).

The two most classical topologies for a SMES are the solenoid and the modular toroid even if other configurations are possible $[15,16]$. Solenoids have higher specific energies than toroids with circular cross section in case of an isotropic conductor [12-14]. Nevertheless, as REBCO tapes behaviour is strongly anisotropic with the $B$ field orientation, a toroidal solution may also be interesting in our case. As the project objective is challenging but the budget dedicated to conductor is limited, it has been necessary to make the best use of the conductor. Two solutions, a solenoid and a torus, have been studied in detail. Their performances are finally rather close and both solutions have advantages and drawbacks.

\subsection{Considerations about magnetic energy storage}

\subsubsection{Virial theorem}

The torus has the advantage of a minimal stray field. The solenoid is minimizing the required amount of conductor in case of an isotropic conductor as well as the amount of associated structural material. The minimum structural mass required to store a magnetic energy $E$ is given by the Virial theorem.

$$
\sigma\left(V_{T}-V_{C}\right)=E \quad \text { or } \quad \frac{\sigma}{\rho}=\frac{E}{M_{T}-M_{C}} .
$$

$\rho$ is the mass density, $\sigma$ is the yield strength of the structural material, $M_{T}$ is the mass in traction, $M_{C}$ is the mass in compression, $V_{T}$ is the volume in traction and $V_{C}$ is the volume in compression of the structural material. The Virial theorem shows that an electromagnet which stores energy is necessarily submitted to stress, and that its volume submitted to tensile stress is higher than its volume submitted to compressive stress. If the winding is selfsupported and the used conductor is the same in the whole system, equation (3) can be applied to the conductor instead of the only structural material. Equation (3) can also be expressed as follows:

$$
\frac{E}{M_{\text {Total }}}=k \frac{\sigma}{\rho}
$$

in which $k$ is a factor depending on the topology. This factor has been calculated for several topologies [12-14]. $k=1 / 3$ for an infinitely thin torus with circular section and $k<1 / 3$ for a real toroid with circular section. $k=1 / 3$ for an infinitely thin and long solenoid. $k>1 / 3$ for a short solenoid. Practically, $k \approx 1 / 2$ is reachable.

\subsubsection{Solenoid pre-design}

In this part, we are considering a solenoid with rectangular cross section with internal radius $R$, thickness $T H$ and height $H$ with homogeneous current density. For a given volume of conductor and a given current density, the maximum energy is obtained for a balanced solenoid in which $R=T H=H$. Nevertheless, this solution generates high magnetic field and is generally not viable for two independent reasons:

- A high $B$ field reduces the current carrying capability of the superconductor and makes the design incompatible with initially assumed current density. The current margin has to be high enough in every point of the coil and is determined by the value and orientation of $B$ at this point and by the $I_{C}(B, \theta)$ curve (see Fig. 3) of the used superconductor.

- The mechanical stress applied to the conductor is too high. This result is linked to the Virial theorem, high specific energy is related to high mechanical stress. The solenoid is submitted to centrifugal forces (radial forces 


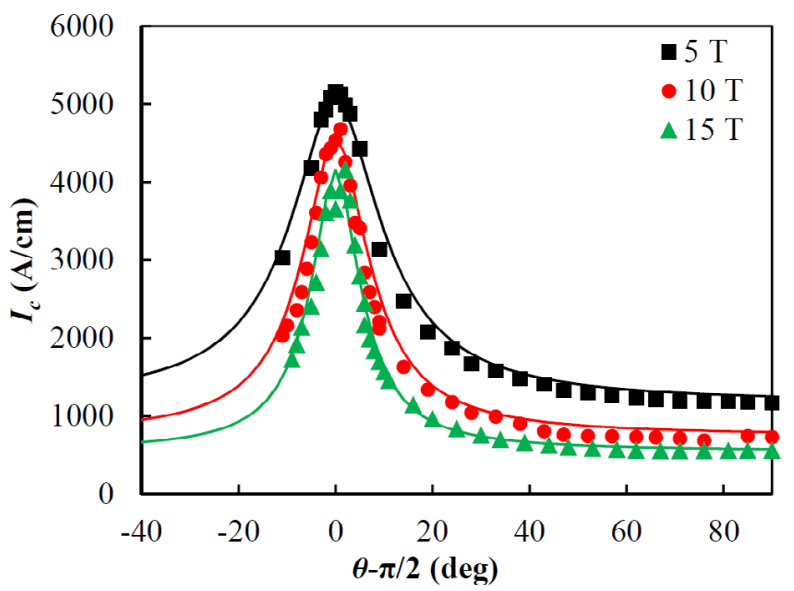

Fig. 3. Critical current of a Fujikura REBCO tape at $4.2 \mathrm{~K}$ with value of $B$ field and its deviation to tape's surface. Data from Tohoku University [17], fit by Fleiter [18].

oriented outwards) on inner turns and centripetal forces (radial forces oriented inwards) on outer turns. This is due to the fact that $B$ is in opposite direction inside and outside the solenoid (and stronger inside than outside the solenoid). This one is also submitted to compressive axial forces. On average, the solenoid is mainly submitted to centrifugal forces and the result is that tapes are submitted to strong azimuthal tensile stress. This is the only stress component useful for energy storage (see Sect. 2.2.1) according to the virial theorem. In the following parts, only this component, called hoop stress, will be considered since the hoop stress is generally the highest stress component to which the winding is submitted. The value of hoop stress is calculated thanks to Wilson's formula [19], in which it is supposed that higher forces applied to inner turns are partially reported on external turns. For the two reasons above, a compromise has to be found, and larger and thinner $(R \gg T H, H \gg T H)$ solenoids are generally preferred.

The specific energy of a solenoid has been calculated for different maximum allowable hoop stresses and different current densities for a total energy of $1 \mathrm{MJ}$ (see Fig. 4). It can be seen that in order to reach high specific energy, high hoop stress and high current density are both necessary. It has to be noticed that these curves are strongly dependent of the considered energy range [20]. In principle, for a solenoid with a mass density $\rho=8900 \mathrm{~kg} / \mathrm{m}^{3}$ (density of copper and Hastelloy ${ }^{\circledR} \mathrm{C}-276$ ), a current density of $500 \mathrm{~A} / \mathrm{mm}^{2}$ and a hoop stress of $300 \mathrm{MPa}$ could be sufficient to reach a specific energy of $20 \mathrm{~kJ} / \mathrm{kg}$. Nevertheless, the anisotropy of REBCO tapes has not yet been taken into account. Some solutions with balanced aspect ratio $(H \approx T H)$ have good specific energy and have high longitudinal field (parallel to tape's surface) which is acceptable for REBCO tapes, but they also have a high transverse field (perpendicular to tape surface). Such a design does not take benefit of REBCO tapes best performances and leads to a high cost of conductor. Figures 5 and 6 show the set of solutions respecting $J=600 \mathrm{~A} / \mathrm{mm}^{2}$ and $\sigma=500 \mathrm{MPa}$ for different aspect ratios $(\alpha=H /(2 R)$ and $\beta=T H / R)$. In order to have a

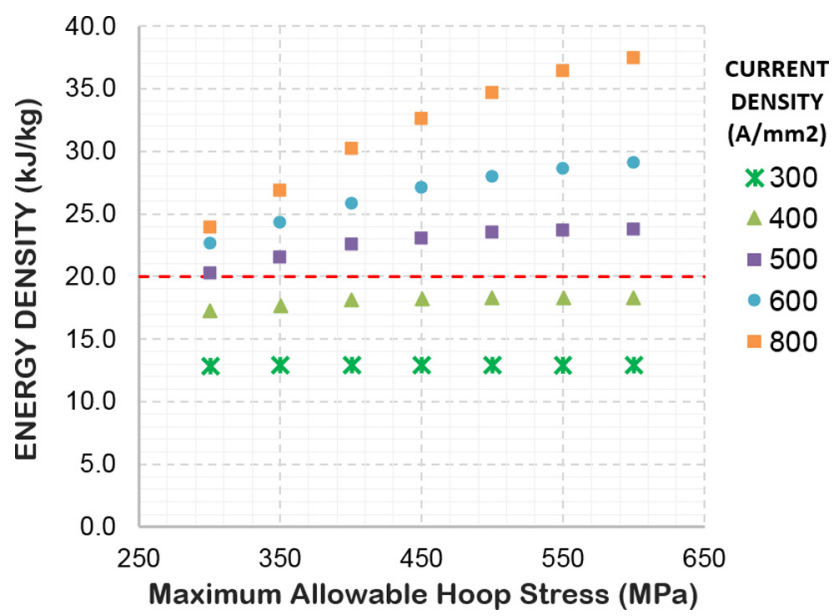

Fig. 4. Maximum possible specific energy as a function of maximum allowable hoop stress and for several current densities $\left(\rho=8900 \mathrm{~kg} / \mathrm{m}^{3}\right), E=1 \mathrm{MJ}$.

more realistic solution, the design tends towards a thinner and higher solution even if this requires even higher current density and hoop stress to reach $20 \mathrm{~kJ} / \mathrm{kg}$. In this case, the transverse field, even if still high, is indeed localized at coil's extremities and it is easier to deal with this problem by working on the coil ends design. That is why a design close to topology 3 is preferred compared to topology 2 (see Fig. 6).

\subsection{Elements of designs comparison}

In the next parts, two very different conceptual designs are compared, a solenoid on one hand and a modular toroid on the other hand. For reasons which will be explained later (see Sect. 2.6), the amount of stabilizer (copper) in the conductor is the same and the conductor is therefore the same in both cases. Its architecture is corresponding to the kind of tape available on the commercial market today at lower price. In both cases, the conductor is a REBCO tape, with a Hastelloy substrate of $60 \mu \mathrm{m}, 30 \mu \mathrm{m}$ of copper stabilizer, and $5 \mu \mathrm{m}$ of silver, buffer and REBCO layer. This bare conductor is surrounded with $20 \mu \mathrm{m}$ of polyimide (insulator) on each side for an average density of $6700 \mathrm{~kg} /$ $\mathrm{m}^{3}$. Most of the commercially available tapes are able to sustain a strain of $0.5 \%$ until they start to lose their superconducting properties [21], which is corresponding in our case to a $500 \mathrm{MPa}$ average stress on the conductor. In both cases, the winding is respecting a mechanical criterion which is to self-support its tensile stress (hoop stress), which is the strongest mechanical stress to which the winding is submitted.

Conductor unit lengths of nearly $200 \mathrm{~m}$ are considered for both designs, because the price per meter of REBCO tapes is stable until this value and increases steeply for longer length due to their manufacturing process. Shorter lengths would lead to more connections and that must be avoided. These connections, which are made by soldering the tapes on copper pieces and/or together, are dissipative and therefore decrease the storage efficiency while increasing the helium consumption. 

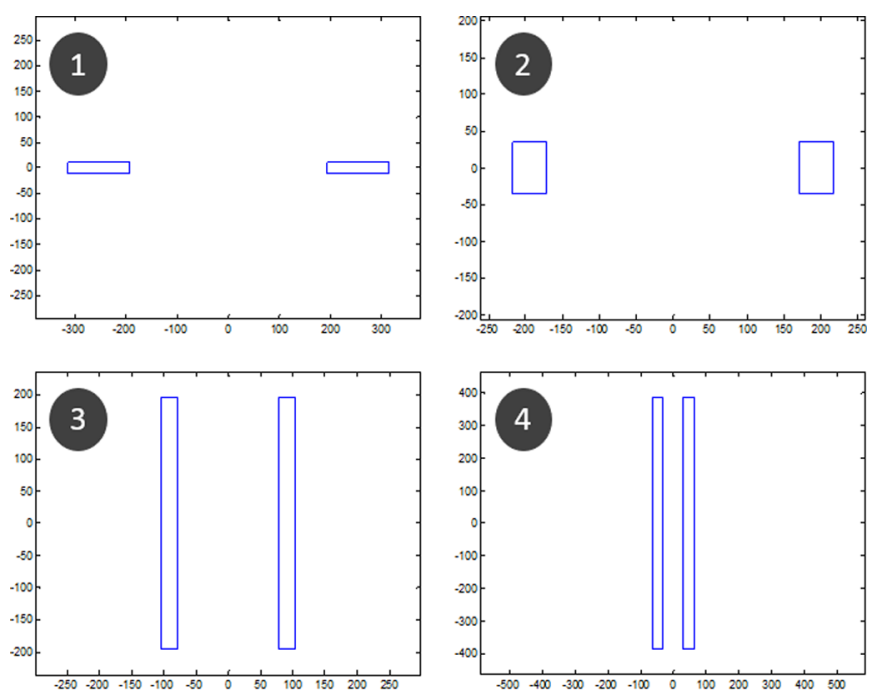

Fig. 5. Cross section view of solenoids 1, 2, 3 and 4 identified in Figure 6. Topology evolution is visible. Axes are graduated in $\mathrm{mm}$.

For the calculation of critical current, we have used the fit made by Fleiter [17,18], based on angular and field dependent measurement of a Fujikura REBCO tape (Fig. 3). Nevertheless, it has to be pointed out that the tape on which these measurements have been done is particularly good and the results obtained may be too optimistic compared with what can be expected on long lengths of tapes of standard quality. That is why in the following designs a large margin between operating current and critical current is considered.

\subsection{Solenoid design}

The designed solenoid has the following characteristics. The inner radius $R$ is $135 \mathrm{~mm}$, the thickness of the section $T H$ is $29 \mathrm{~mm}$ and the total height $H$ is $384 \mathrm{~mm}$. It is made up of 42 pancakes, a pancake being a unit length winding. The complete winding process is as follows: a massive piece of copper, called internal contact, is fixed in a mandrel. The superconducting tape is soldered on the internal contact and then wound until the external radius is reached. The superconducting tape is then soldered on the external side of a crescent-moon-shaped massive piece of copper, called external contact. A few additional turns are wound after this contact then glued together with Stycast ${ }^{\circledR}$ glue, which is adapted to cryogenic temperatures, in order to finalize the pancake. The different pancakes are then connected together at the internal and external contacts (see Fig. 7).

Each pancake has the same inner radius and thickness and is a 214 turns winding of a $200 \mathrm{~m}$ tape length, but pancakes are made of tapes with different widths. Fourteen pancakes are $4 \mathrm{~mm}$ wide, 10 pancakes are $6 \mathrm{~mm}$ wide, 16 pancakes are $8 \mathrm{~mm}$ wide and 2 pancakes are $12 \mathrm{~mm}$ wide. The pancakes of $4 \mathrm{~mm}$ are in the central part of the solenoid and then the thickness of pancakes is increased until the end of the solenoid. It is called a graded magnet design. Enlarging the width of the tapes aims to compensate the reduction of critical current due to higher transverse field in the solenoid extremities and then to supply all pancakes in serial.

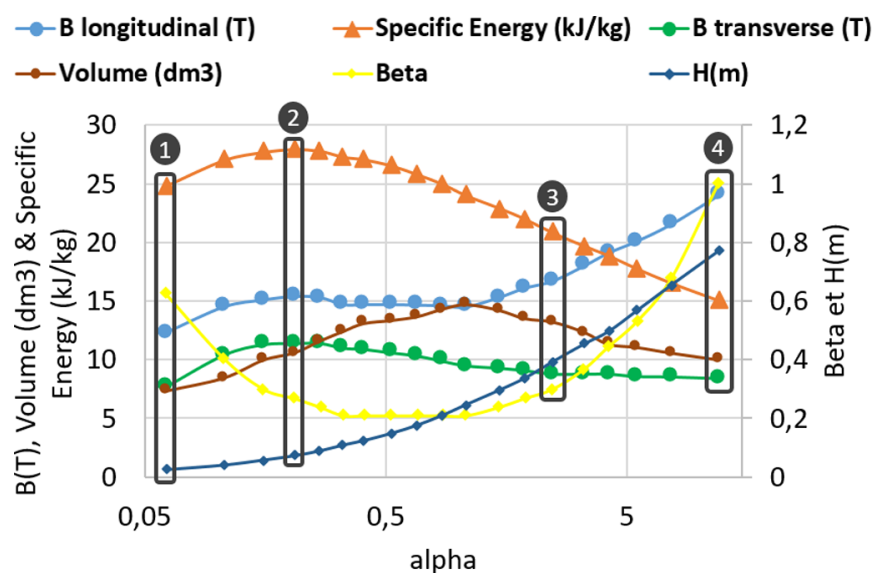

Fig. 6. Set of topological solutions respecting $E=1 \mathrm{MJ}, \sigma=500$ $\mathrm{MPa}$, and $J=600 \mathrm{~A} / \mathrm{mm}^{2}$ for different values of aspect ratio $\alpha$. Solutions at the left are corresponding to flat solenoids, and solutions on the right are very long solenoids. $B$ longitudinal is the maximum $B$ field in mid-plan of the coil and $B$ transverse is the maximum radial $B$ field (at the top and middle of coil's section). $H$ is the height.

The pancakes are separated by flanges of G10, which is a standardized glass-reinforced epoxy laminate. G10 was originally developed for electronics and is widely used in cryogenic electrotechnics due to its good electrical insulating qualities, mechanical strength and well characterized thermal extension coefficients. The mandrels are also made of G10. The thickness of the flanges are tuned in order to control the value of transverse field in different pancakes and thus to homogenize the critical current along the coil. The maximum field is $10.8 \mathrm{~T}$ and the maximum transverse field is $5.1 \mathrm{~T}$. Engineering current density is $700 \mathrm{~A} / \mathrm{mm}^{2}$ in the $4 \mathrm{~mm}$ wide pancakes. Operating current is $380 \mathrm{~A}$ and critical current is $1270 \mathrm{~A}$; thus, operating current is $30 \%$ of $I_{C}$. Hoop stress is $484 \mathrm{MPa}$. Energy is $943 \mathrm{~kJ}$. The inductance is $13.3 \mathrm{H}$. Specific energy is $19.4 \mathrm{~kJ} /$ $\mathrm{kg}$. Expressed as an equivalence of $4 \mathrm{~mm}$ wide conductor, the total length is $13.4 \mathrm{~km}$. The total volume of conductor is $7.24 \mathrm{dm}^{3}$ (see Fig. 8).

\subsection{Modular toroid design}

In the solenoidal solution, the current carrying capacity is reduced by the transverse field at coil's extremities. The total specific energy is reduced because of the wider tapes used to ensure a high operating current. This problem is avoided in a toroid. There is indeed no edge effect and the $B$ field is purely longitudinal in a perfect torus. Consequently, a toroidal solution has been studied and the optimal design obtained is described below.

Our solution is a modular toroid with D-shaped section. Such a D-shaped section (see Fig. 9) is preferable for a compact toroid. Inside a torus, the $B$ field evolves inversely proportional with the distance to torus central axis. A perfect Princeton-D shape section makes the tension regular all along the conductor [19]. It also improves specific energy compared to a circular cross section. This last fact seems in agreement with Virial theorem and has 


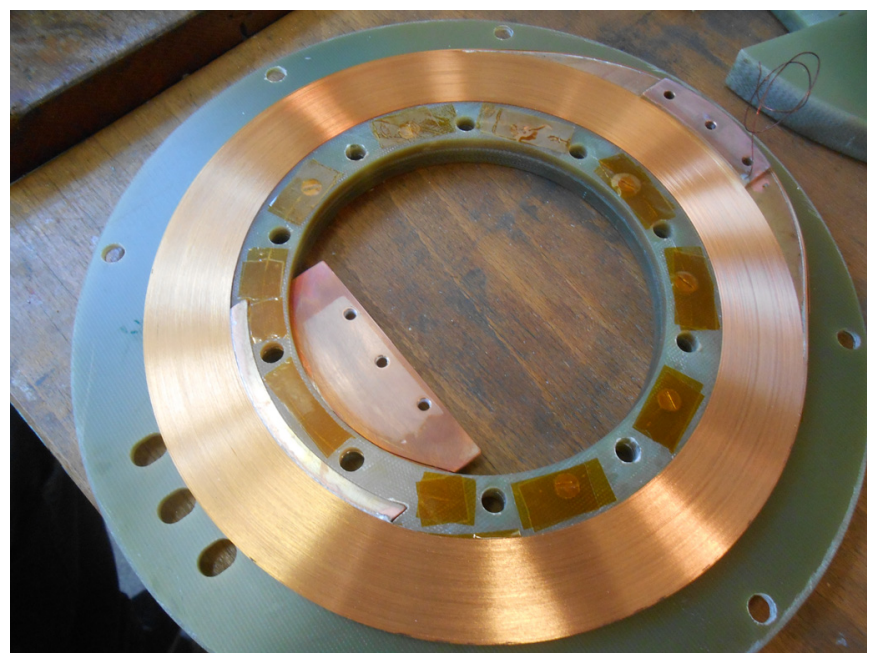

Fig. 7. View of a prototype pancake. The mandrel, the internal contact, the winding, the external contact and a flange are visible.

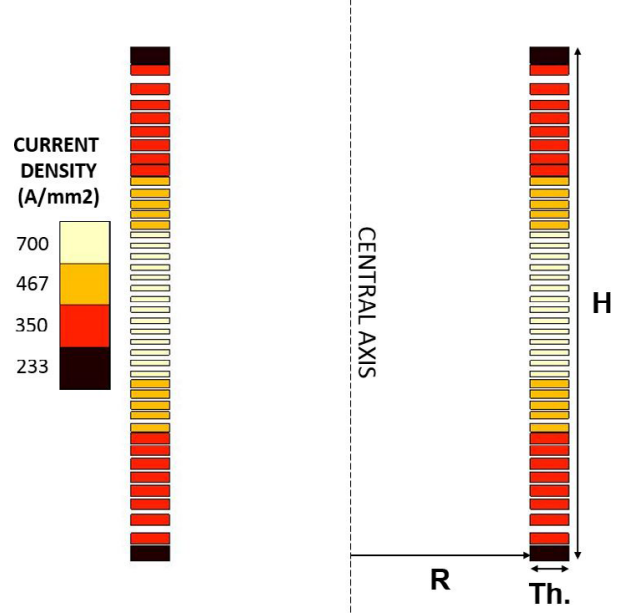

Fig. 8. Cross section view of the solenoid. As the tapes are wider, the current density is lower at solenoid extremities. $R=135 \mathrm{~mm}$, $T H=29 \mathrm{~mm}, H=384 \mathrm{~mm}$.

been demonstrated [22]. We have found the same result when comparing D-shape and circular cross section solutions with finite element software Flux ${ }^{\circledR}$ [23]. Nevertheless, the right part of the D-shape coil needs to lean on an external structure (central trunk).

Design characteristics are as follows (see Fig. 10): the total diameter is $580 \mathrm{~mm}$ for a total height of $360 \mathrm{~mm}$. The modular toroid is made of 112 single pancakes, i.e. 56 double pancake. Unit length of a double pancake is $193 \mathrm{~m}$. All pancakes are made of $4 \mathrm{~mm}$ wide tape and are arranged symmetrically around the torus central axis, i.e. with an angle of $3.2^{\circ}$ between each single pancake. This enables to reduce the maximum transverse field to $1.6 \mathrm{~T}$, while the maximum longitudinal field is $10.3 \mathrm{~T}$. Engineering current density is $915 \mathrm{~A} / \mathrm{mm}^{2}$. Operating current is $500 \mathrm{~A}$ and critical current $I_{C}$ is $1440 \mathrm{~A}$; thus, operating current is $35 \%$ of $I_{C}$. Hoop stress is $450 \mathrm{MPa}$. Centering force on a single pancake is $50 \mathrm{kN}$. Total energy is $952 \mathrm{~kJ}$, inductance is

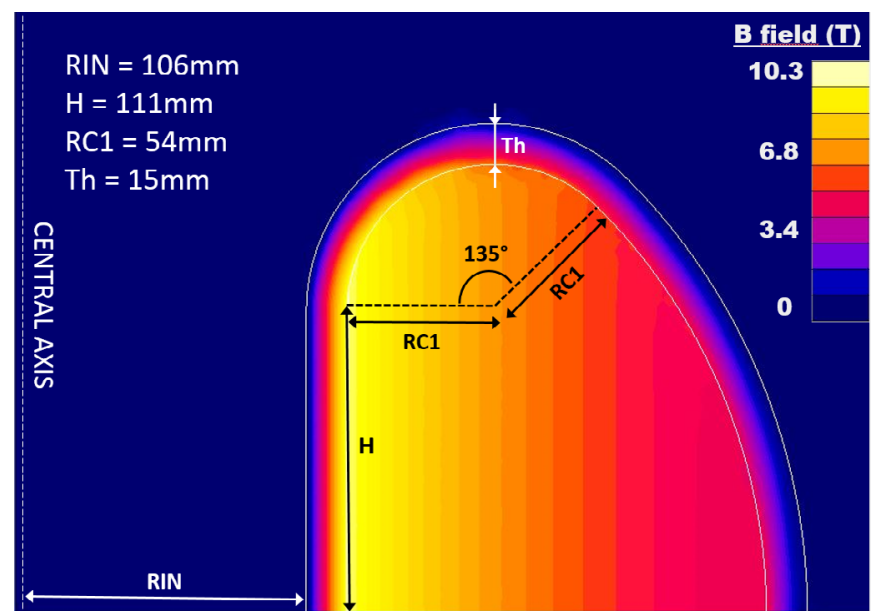

Fig. 9. View of a half D-shape section. The magnetic field is 0 outside the torus. Inside the torus, $B$ is decreasing inversely proportional with the distance to torus central axis.

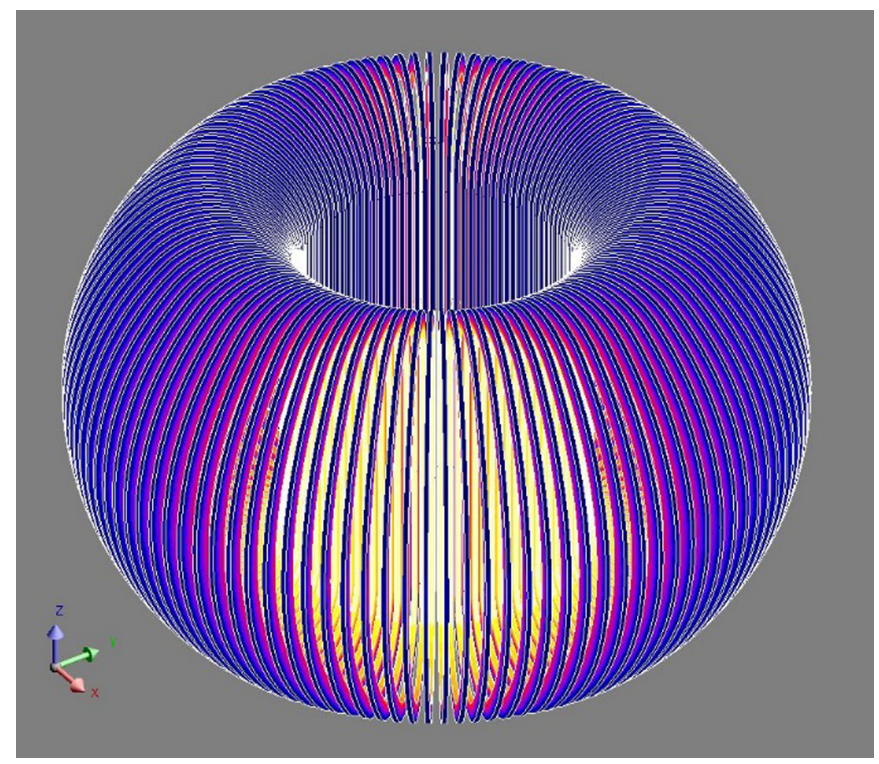

Fig. 10. View of the complete modular toroid, with 112 single pancakes.

$7.8 \mathrm{H}$. Specific energy is $24.7 \mathrm{~kJ} / \mathrm{kg}$. Total length of conductor is $10.6 \mathrm{~km}$. The total volume of conductor is $5.72 \mathrm{dm}^{3}$, which is $21 \%$ less than for the solenoid.

\subsection{Comparison between torus and solenoid}

From the results above, the following conclusion can be drawn. At first sight, it seems clear that the toroidal version is better both from the budget and specific energy points of view. However, as a reminder, our design is based on properties of a Fujikura tape. Fujikura tapes are very anisotropic, and their critical current is 6 times higher in longitudinal field than in transverse field at $10 \mathrm{~T}$. This is not necessarily the case for tapes from all manufacturers. That is why considering the $J(B)$ properties of another manufacturer's tape, SuperOx [24], may lead to the opposite conclusion, in which a solenoid would require less conductor than a toroid. 
Table 1. Performances comparison of the two designs.

\begin{tabular}{|c|c|c|}
\hline Nominal values & Solenoid & Toroid \\
\hline Energy $(\mathrm{kJ})$ & 950 & 950 \\
\hline External diameter $(\mathrm{cm})$ & 33 & 58 \\
\hline Height (cm) & 38 & 36 \\
\hline Cryostat volume $\left(\mathrm{dm}^{3}\right)$ & 32 & 94 \\
\hline$B_{/ / \max }(\mathrm{T})$ & 10.8 & 10.3 \\
\hline$B_{T} \max (\mathrm{T})$ & 5.1 & 1.6 \\
\hline$I_{\text {OPERATING }}(\mathrm{A})$ & 380 & 500 \\
\hline$I_{\text {OPERATING }} / I_{\text {CRITICAL }}$ (Fujikura) & $30 \%$ & $35 \%$ \\
\hline$I_{\text {OPERATING }} / I_{\text {CRITICAL }}($ SuperOx $)$ & $37.5 \%$ & $66 \%$ \\
\hline$J_{\text {conductor }} \max \left(\mathrm{A} / \mathrm{mm}^{2}\right)$ & 700 & 915 \\
\hline Hoop stress $(\mathrm{MPa})$ & 484 & 450 \\
\hline Inductance $(\mathrm{H})$ & 13.3 & 7.8 \\
\hline Volume of conductor $\left(\mathrm{dm}^{3}\right)$ & 7.24 & 5.72 \\
\hline Specific energy (kJ/kg) & 19.4 & 24 \\
\hline
\end{tabular}

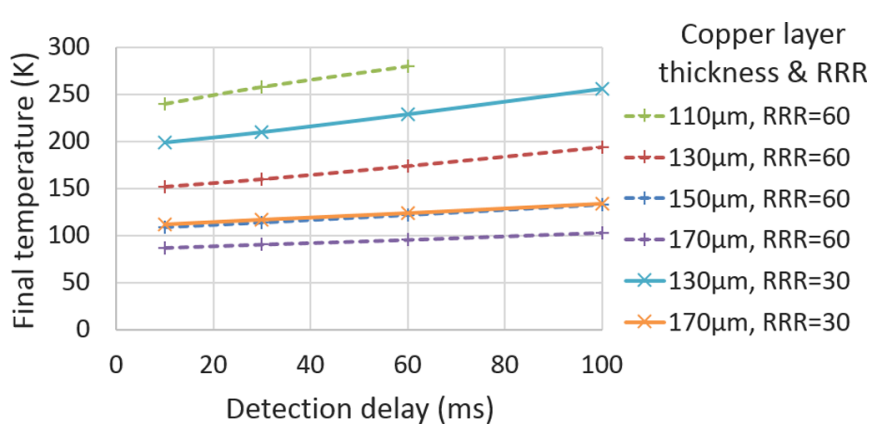

Fig. 11. Maximal hotspot temperature as a function of detection delay for different copper thicknesses and different RRR.

In Table 1, the two designs described in Sections 2.3 and 2.4 are compared. If the current margin is calculated again by considering SuperOx tapes instead of Fujikura tapes, operating current of the toroid is now $66 \%$ of critical current. This value is too high given our protection strategy (see Sect. 2.6).

Other considerations have to be taken into account. - Of course, the toroid has the great advantage to have a minimal stray field. It is also easier to divide the toroid in several stages and to discharge the coils in parallel thanks to an XRAM [25] system. The term "XRAM" generator comes from the term "Marx" generator spelled backwards since the XRAM generator principle consists in charging several inductances in series and then discharging them in parallel, thus multiplying the output current by the number of stages whereas the Marx generator principle consists in charging several capacitors in parallel and then discharging them in series, thus multiplying the output voltage.

- If we consider cylindrical cryostats, the solenoid's cryostat has an inner volume of $32 \mathrm{dm}^{3}$ and the toroid's one a volume of $94 \mathrm{dm}^{3}$. The solenoid is therefore more compact.
- A last fact is that the solenoid is much simpler to build. In toroid, small distances and small angles could increase the price of machining structure pieces. The pancakes of the solenoid are also much easier to test in a field generated by a solenoidal magnet.

Finally in the frame of BOSSE project, a solenoidal SMES will be manufactured for two independent reasons: first, as written above, because it is much more difficult and costly to machine the structural elements separating the pancakes and second, because SuperOx was the only supplier whose sales offer was satisfying our conductor budget for the required volume of conductor. In the topologies presented Table 1 the use of SuperOx tapes reduces too much the current margins for the toroid design. It is interesting to notice that in the end, the suppliers' tape properties are governing the design choices in our case, but for practical applications in which the stray field would be problematic and high output current necessary, a torus would have non-negligible advantages and could be made using other tapes.

\subsection{Protection}

The current density and hoop stress in bare conductor without insulation are $1000 \mathrm{~A} / \mathrm{mm}^{2}$ and $690 \mathrm{MPa}$ in central pancakes of the solenoid and $1300 \mathrm{~A} / \mathrm{mm}^{2}$ and $640 \mathrm{MPa}$ in the toroid. So high current density and mechanical stress are rarely found together in a superconducting coil.

In Low Temperature Superconductors (LTS) windings (i.e. niobium-titanium or niobium-tin), some very small input of energy such as conductor's small displacements, small cracks of impregnation resin or even vibrations can lead to a loss of the superconducting state. This is due to the very small temperature margin of LTS (a few kelvin), and to the low heat capacities of materials at very low temperature [1]. The beneficial aspect of this small temperature margin is that a local transition to normal 


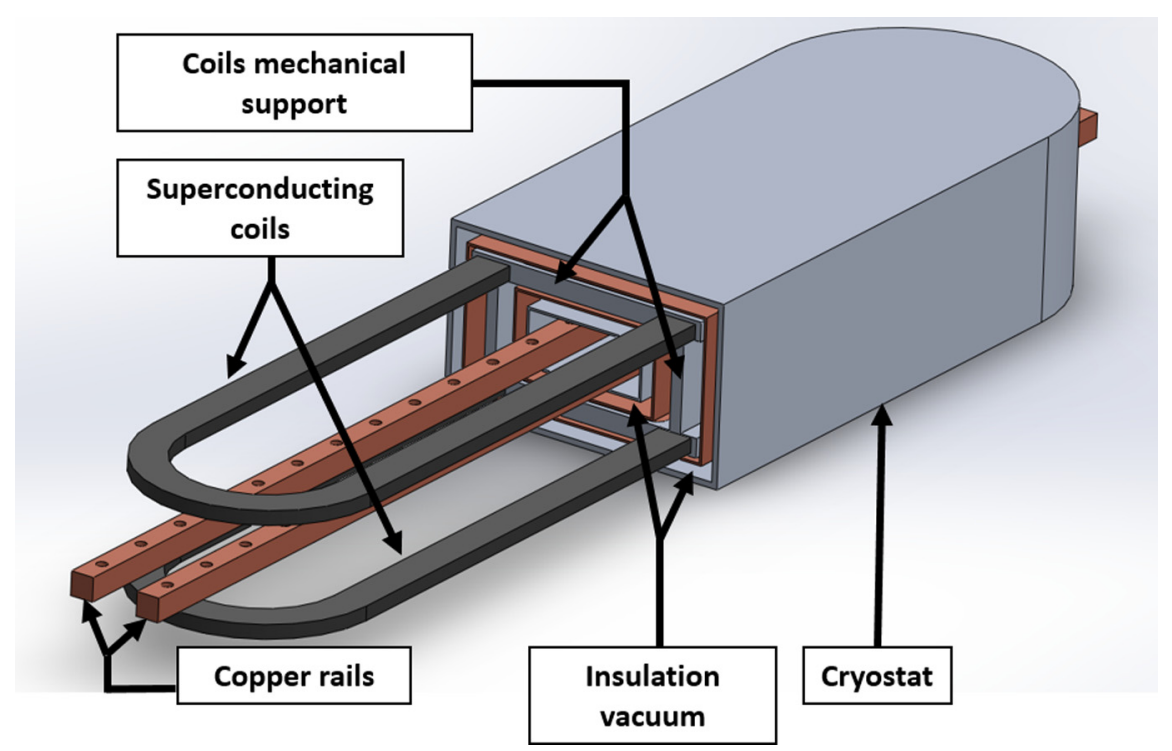

Fig. 12. CAO plan of the S3EL launcher (courtesy R. Pasquet, SigmaPhi).

state quickly propagates in the winding. A detection by voltage measurement is therefore possible before the conductor is damaged by local temperature rise. LTS are stabilized by copper or aluminium in order to avoid thermal runaway due to small energy inputs. A large amount of stabilizer is also useful to limit the temperature rise when a global thermal avalanche happens. In this kind of event, called a "quench", a large part of the coil or the whole coil is transited to resistive state. In this case, the coil is discharged in an external resistor, so the time constant depends on the inductance and the discharge voltage. Preferentially, the temperature is kept under $100 \mathrm{~K}$ to avoid localized thermal dilatation and thus dangerous mechanical stress.

In the case of the aluminium stabilized Nb-Ti BESS coil (Balloon-Borne Experiment with a Superconducting Solenoid Magnet Spectrometer) [26] which currently owns the specific energy world record for a superconducting coil, the aluminium plays a role both for stabilization and mechanical structure [27]. In the case of standard REBCO tapes unfortunately, Hastelloy ${ }^{\circledR}$ or other NiW alloys are used for the substrate, and they are very poor electrical conductors. Copper is used as stabilizer but adds negligible mechanical reinforcement. Furthermore, the $1 \mathrm{MJ}$ range leads to rather large inductances unless using multi-tape cables $[9,10]$, which would add another layer of complexity and was not considered in this project. Such high inductance slows down the coil discharge. If a loss of the superconducting state is supposed at instant $t=0$, the temperature increase can be calculated as a function of the event detection delay, of the discharge speed (linked to coil's voltage) and conductor's thermal capacitance and electrical resistance. The maximum temperature has been evaluated with a very simple adiabatic 0-D hot spot model for different detection times (see Fig. 11). On our test bench dedicated to short samples measurement, the detection delay is between 30 and $60 \mathrm{~ms}$. Several curves are drawn depending on the copper thickness and for two different values of RRR (Resistive Residual Ratio). The RRR is the ratio between resistivity at room temperature and resistivity at $4.2 \mathrm{~K}$. The RRR of copper deposited on REBCO tapes (by electrodeposition or lamination) practically does not overtake 60 and is generally lower. Calculations are made with a discharge voltage of $5 \mathrm{kV}$. We can see that if we are considering the same standard protection criterion that was applied for LTS coils, a copper stabilizing thickness of more than $170 \mu \mathrm{m}$ would be necessary. This value is incompatible with the $20 \mathrm{~kJ} / \mathrm{kg}$ criteria.

Another logic has therefore to be followed in order to ensure safety of the coil at operating current. The REBCO tapes in liquid helium have the advantage to be very stable [28] due to their high temperature margin. Contrary to LTS, they have high Minimum Quench Energy and no loss of superconducting state can be caused by another event that overtake the critical current. We therefore assume that a sufficient margin between operating and critical current will guarantee the safety of the coil. The difficulty lies in defining exactly what the required margin is. Indeed, a major issue of REBCO wires is the inhomogeneity of their performances along the length. Performance variation occurs even at very small scale [29]. Influence of small-scale defects on global critical current regarding to stabilizer thickness needs to be investigated further. Furthermore, the statistical distribution of these defects is not known at $4.2 \mathrm{~K}$ by lack of experimental data.

Practically in BOSSE project, an important stage will be to determine maximum performance of each pancake tested individually under magnetic field provided by an external magnet. The small conductor length and low inductance of a pancake compared to complete SMES will facilitate the detection of a transition (criterion $1 \mu \mathrm{V} / \mathrm{cm}$ ) and the quick discharge of the element under high voltage $(5 \mathrm{kV})$.

It is a noteworthy fact that by using a standard tape with a large current margin, we are decorrelating the amount of stabilizer from the operating current of the SMES. Following this reasoning, the objective to reach high specific energy becomes the same as reducing the total length of conductor. 


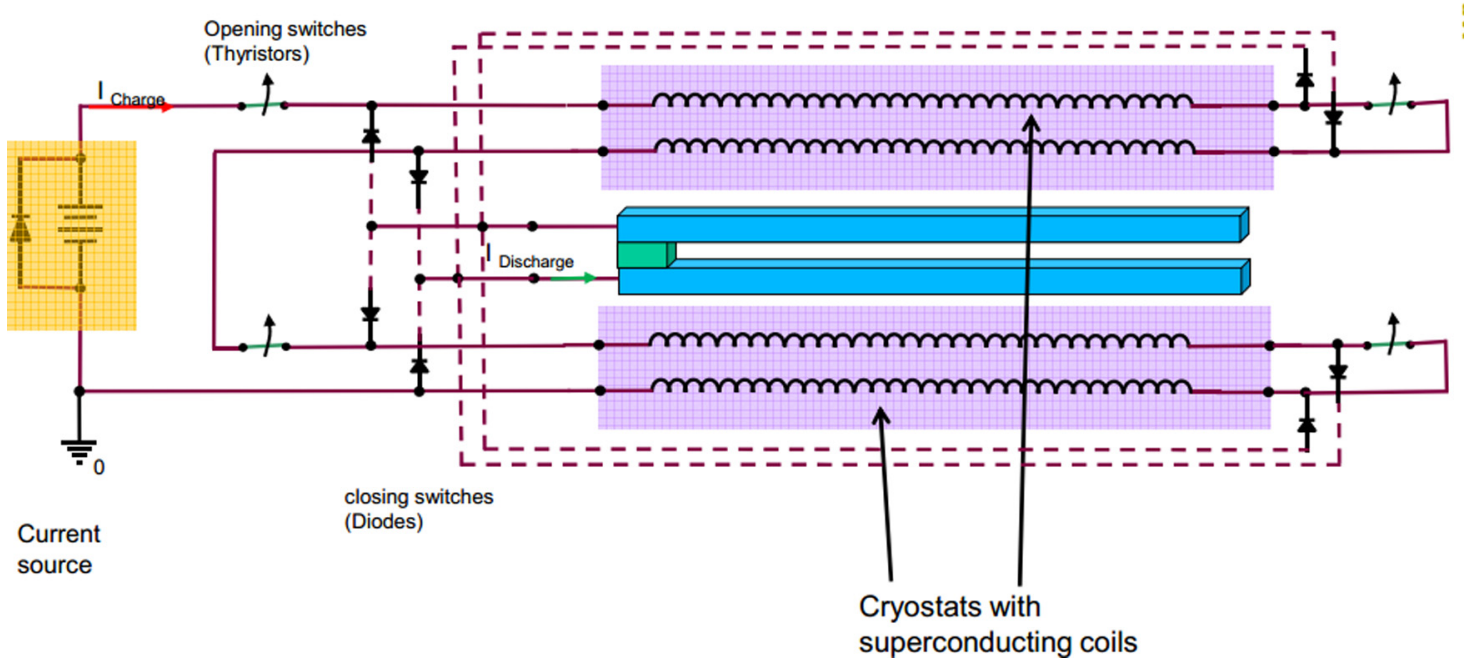

Fig. 13. Principle diagram of the launcher electrical circuit (courtesy V. Brommer, ISL).

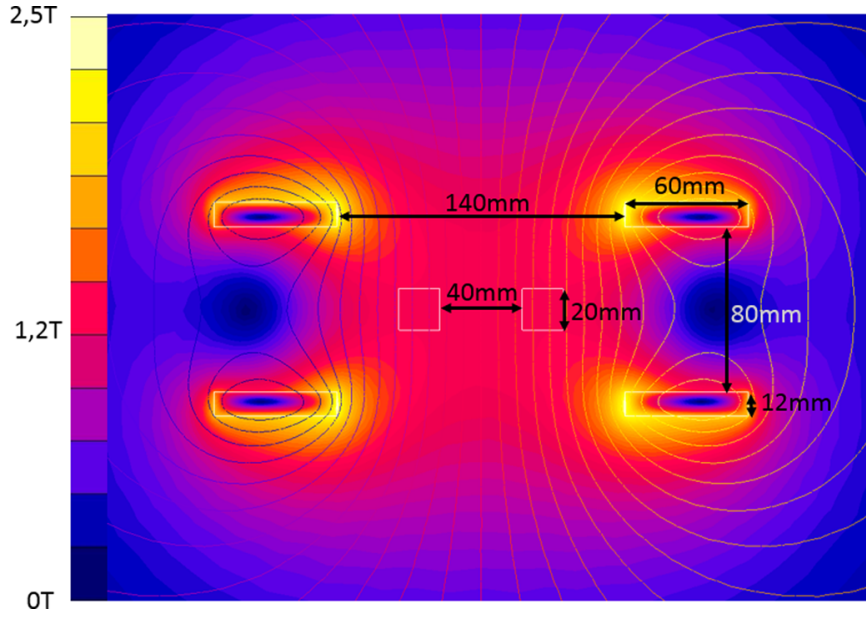

Fig. 14. Cross section view of induction field when the coil is charged, just before launching. The field between the rails is then $1.2 \mathrm{~T}$. At the beginning of the launch, the field rises until $1.4 \mathrm{~T}$ (additional field generated by current the rails).

\section{Electromagnetic launcher S3EL}

As mentioned in Section 1, the second objective of BOSSE project is to manufacture and test a low scale prototype of S3EL concept. The prototype will be $1 \mathrm{~m}$ long and will accelerate a $60 \mathrm{~g}$ projectile to $100 \mathrm{~m} / \mathrm{s}$ (see Fig. 12). An iterative work has been leaded to position the superconducting coils by taking into account the space needed for mechanical support of the rails and for the cryostat and to find the best compromise between stored energy, rails/ coils coupling, value of $B$ field on the conductor and conductor's current. The current in the rails is $12 \mathrm{kA}$ for $3 \mathrm{kA}$ in the conductor, multiplied by a four stages XRAM system [25] (see Fig. 13). The induction field is nearly $1.4 \mathrm{~T}$ between the rails during the shot and $2.5 \mathrm{~T}$ on the coils (Fig. 14). Nominal current is nearly $45 \%$ of critical current. The high current conductor is made of two $12 \mathrm{~mm}$ wide

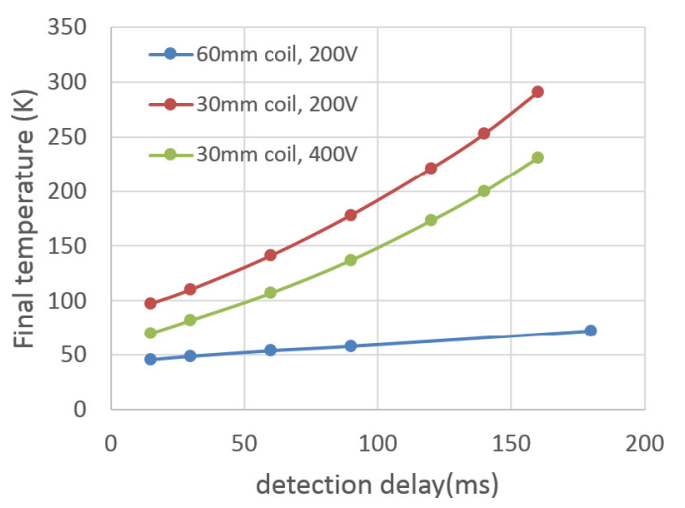

Fig. 15. Maximal hotspot temperature as a function of detection delay, in two different configurations (total thickness of the winding $=30$ or $60 \mathrm{~mm}$ ) and different discharge voltage. $\mathrm{RRR}=15$.

REBCO tapes soldered face-to-face. The two coils (upper coil and lower coil) are each made of two co-winded subcoils in order to constitute the four equivalent stages of the launcher. Each of sub-coil is made of a superconducting conductor (nearly $200 \mu \mathrm{m}$ thick) and a stabilizer made of copper, insulated on one side (nearly $800 \mu \mathrm{m}$ thick). Four elements are therefore co-winded to constitute one coil. This winding method is the same as in the EUCARD project [30]. Each sub coil has 30 turns.

Contrary to the EUCARD REBCO dipole developed for particle accelerators, there is no external geometric constraint for BOSSE launcher. A dipolar racetrack topology with very wide and flat section $(60 \mathrm{~mm} \times 12 \mathrm{~mm})$ has been chosen in order to minimize the transverse field seen by the conductor. This also allows to greatly stabilize the coil ( $80 \%$ of copper in the section), contrary to the strategy adopted for the solenoidal SMES. This ensures protection of the coil despite a discharge voltage limited to $200 \mathrm{~V}$ (Fig. 15). The turn-to-turn voltage has to be limited because the co-winding of four elements prevents to use an insulator surrounding each conductor. 


\section{Conclusions}

The BOSSE project has two ambitious objectives: to build a solenoidal SMES which will exceed the world record of specific energy for a superconducting coil and to build the first proof of concept of the S3EL electromagnetic launcher. These two objectives will be reached thanks to the use of last generation superconductors called REBCO. The design of these two prototypes is essentially determined by the REBCO tapes specific features and is focused on taking advantage of their unique properties. The two prototypes will be built and tested by December 2018 . BOSSE project is part of a strong technological development around REBCO HTS tapes. It has a synergy with other projects such as very high field superconducting magnets (NOUGAT project) [22] or high field HTS dipole magnets considered for the future of the Large Hadron Collider in CERN (projects EUCARD et EUCARD-2) $[10,30]$.

This work is funded by French DGA in Project BOSSE.

\section{References}

1. P. Tixador, Les supraconducteurs (Edition Hermès, 1995)

2. C.A. Luongo, IEEE Trans. Magn. 32, 2214 (1996)

3. P. Tixador, B. Bellin, M. Deleglise, J.C. Vallier, C.E. Bruzek, S. Pavard, J.M. Saugrain, IEEE Trans. Appl. Supercond. 17, 1707 (2007)

4. R.T. Meyer, R.A. DeCarlo, J. Dickerson, IEEE Trans. Plasma Sci. 45, 702 (2017)

5. G.-H. Wang et al., IEEE Trans. Plasma Sci. 44, 1424 (2016)

6. P. Lehmann, H. Peter, J. Wey, IEEE Trans. Magn. 37, 435 (2001)

7. A. Badel, P. Tixador, M. Amiet, V. Brommer, IEEE Trans. Appl. Supercond. 22, 5700204 (2012)

8. A. Badel, P. Tixador, M. Amiet, Supercond. Sci. Technol. 25, 014006 (2012)

9. D.C. Van der Laan, P.D. Noyes, G.E. Miller, H.W. Weijers, G.P. Willering, Supercond. Sci. Technol. 26, 045005 (2013)

10. L. Rossi, A. Badel, M. Bajko, A. Ballarino, L. Bottura, M.M.J. Dhalle, M. Durante, Ph. Fazilleau, J. Fleiter, W. Goldacker, E. Haro, IEEE Trans. Appl. Supercond. 25, 1 (2015)

11. A. Badel, P. Tixador, P. Dedié, IEEE Trans. Appl. Supercond. 21, 1375 (2011)
12. I.N. Sviatoslavsky, W.C. Young, Nucl. Eng. Des. 58, 207 (1980)

13. F.C. Moon, J. Appl. Phys. 53, 9112 (1982)

14. W. Hassenzahl, IEEE Trans. Magn. 25, 1799 (1989)

15. S. Nomura, T. Osaki, J. Kondoh, H. Tsutsui, S. Tsuji-Iio, Y. Sato, R. Shimada, IEEE Trans. Appl. Supercond. 9, 354 (1999)

16. O. Vincent-Viry, A. Mailfert, D. Trassart, IEEE Trans. Appl. Supercond. 11, 1916 (2001)

17. S. Fujita, M. Daibo, M. Igarashi, R. Kikutake, K. Kakimoto, Y. Iijima, M. Itoh, T. Saitoh, J. Phys.: Conf. Ser. (IOP Publishing) 507, 022007 (2014)

18. J. Fleiter, Study of the implementation of high temperature superconductors in accelerator magnets, Ph.D. thesis, University of Grenoble, 2013

19. M. Wilson, Superconducting Magnets (Clarendon Press, Oxford, UK, 1983)

20. B. Vincent, P. Tixador, T. Lecrevisse, J.-M. Rey, X. Chaud, Y. Miyoshi, IEEE Trans. Appl. Supercond. 23, 5700805 (2013)

21. C. Barth, G. Mondonico, C. Senatore, Supercond. Sci. Technol. 28, 045011 (2015)

22. I. Mazurenko, A. Pavlyuk, Y. Vasetsky, Przegląd Elektrotechniczny 88, 67 (2012)

23. http://www.cedrat.com/

24. T. Benkel, G. Escamez, A. Badel, X. Chaud, D. Gonzales, P. Tixador, P. Fazilleau, T. Lécrevisse, IEEE Trans. Appl. Supercond. 26, 1 (2016)

25. P. Dedie, V. Brommer, A. Badel, P. Tixador, IEEE Trans. Dielectr. Electr. Insul. 18, 1189 (2011)

26. A. Yamamoto, Y. Makida, H. Yamaoka, H. Ohmiya, K. Tanaka, T. Haruyama, T. Yoshida, K. Yoshimura, S. Matsuda, K. Kikuchi, Y. Ootani, S. Mizumaki, IEEE Trans. Appl. Supercond. 12, 438 (2002)

27. K. Wada, S. Meguro, H. Sakamoto, T. Shimada, Y. Nagasu, I. Inoue, K. Tsunoda, S. Endo, A. Yamamoto, Y. Makida, K. Tanaka, Y. Doi, T. Kondo, IEEE Trans. Appl. Supercond. 10, $373(2000)$

28. Y. Miyoshi, A. Badel, X. Chaud, T. Benkel, B. Vincent, P. Tixador, J. Marpaud, F. Debray, T. Lecrevisse, M. Devaux, P. Fazilleau, J.-M. Rey, IEEE Trans. Appl. Supercond. 25, 1 (2015)

29. K. Higashikawa, K. Katahira, M. Inoue, T. Kiss, Y. Shingai, M. Konishi, K. Ohmatsu, T. Machi, M. Yoshizumi, T. Izumi, Y. Shiohara, IEEE Trans. Appl. Supercond. 24, 1 (2014)

30. G. de Rijk, IEEE Trans. Appl. Supercond. 22, 4301204 (2012)

Cite this article as: Jérémie Ciceron, Arnaud Badel, Pascal Tixador, Superconducting magnetic energy storage and superconducting self-supplied electromagnetic launcher, Eur. Phys. J. Appl. Phys. 80, 20901 (2017) 